Föderalisation (S. 29) des gesamten Bundesgebietes war abgeschlossen, die Territorien als Bundesreserve für das Zurverfügungstellen von neuen Gliedstaaten erschöpft. Die Etablierung der erforderlichen Organe und gesetzlichen Grundlagen wurde von dem Interimsgouverneur eingeleitet. Mit den Wahlen vom 10. November 1974 zur Verfassungsgebenden Versammlung (zugleich mit den Gemeinde- und a. O. Bundesdeputierten- und Senatorenwahlen) hatte die Bevölkerung Gelegenheit, sich auf diesem Wege als Staatsvolk zu konstituieren und zu artikulieren.

Hierbei soll nur die Frage gestellt, aber nicht beantwortet werden, was es ist, was eine Bevölkerung eines Gebietes, zu dem sie keine weitere Beziehung hat als dort zu wohnen, eigentlich zu dessen Staatsvolk macht, ob nicht als Essential das entsprechende Bewußtsein hinzukommen muß? Diese Wahlen jedenfalls sprechen nicht gerade für einen überwältigenden Wunsch nach eigener Staatlichkeit und vermochten daher den Geburtsfehler eines vom Bund eingehauchten Lebens nicht zu beheben. Von den 74017 Wahlberechtigten der Bevölkerung von mehr als 150000 (S. 36) beteiligten sich nur $34956=$ $47,22 \%$. Von den gültigen Stimmen erhielt der PRI 87,4\%.

Die Verfassung des neuen Staates vom 9. Januar 1975 (abgedruckt bei Lucero A., S. 117-158) $)^{2}$ hält sich in den engen Grenzen des von der Bundesverfassung Vorgegebenen, so daß diese Úbereinstimmung sicherlich $70 \%$ des Textes umfaßt (S. 86).

Der Vorzug der hier angezeigten Arbeit liegt darin, daß vor allem für den Außenstehenden ein Blick in die Institutionengeschichte der wissenschaftlich etwas vernachlässigten Einzelstaaten gegeben wird (hierauf weist Fix-Zamudio in seinem Vorwort hin, S. 13) einschließlich des bereits erwähnten Zahlenmaterials aus den maßgeblichen Wahlen. Politische und soziologische Aspekte fehlen indes völlig.

Gerhard Scheffler

\title{
Waldemar Hummer
}

Revindikation von historischen Gebietstiteln in Lateinamerika - Die Forderung Boliviens auf Zugang zum Meer

Kleine Arbeitsreihe zur Europäischen und Vergleichenden Rechtsgeschichte, Heft 16, Verlag Druck Schodl Weiz, Graz, 1983, 72 S., 100 Schilling

Fast wäre es ein historischer Zufall gewesen, der Bolivien den langersehnten Zugang zum Pazifik wiedergegeben hätte. Als Peru und Chile 1926 bezüglich ihrer konkurrierenden Gebietsansprüche über die Gegend von Tacna und Arica zu keinem Ausgleich finden konnten, wurde erwogen, dieses streitverfangene Territorium einfach an Bolivien zu übertragen, um so den Konflikt zu lösen. Im Verlaufe der Vermittlungsbemühungen von

2 Vgl. weiter die offizielle Sammlung der Verfassungstexte durch die Secretería de Gebernación, México, v. 5. 2. 1980 , Bd. I, S. 147-185. 
US-Außenminister Kellogg konnten beide Parteien dann aber doch noch zu einem Ergebnis gelangen. Der Vertrag von Lima (3. 6. 1929) schlug die Gegend um Tacna Peru und die Hafenstadt Arica Chile zu. Spätere Zessionen dieser Gebiete wurden von der Zustimmung des jeweils anderen Vertragspartners in einem Zusatzprotokoll abhängig gemacht. Bolivien verblieb bis heute im Zustand eines "landlocked state".

Für geschichtliche Fußnoten dieser Art ist in der Arbeit von Waldemar Hummer kein Raum. Der Autor nutzt die erste Hälfte seiner Betrachtung, um die historischen Gegebenheiten, die zu Boliviens Binnenlage geführt haben, überblickhaft darzustellen. Er tut dies in zusammenfassender Form und führt damit auch den Leser, der nicht mit den spezifischen Territorialkonflikten Lateinamerikas vertraut ist, gut an die Problemlage heran. Dabei beläßt es Hummer nicht nur bei den rein rechtserheblichen Fakten, sondern er streift auch die politischen und sozio-ökonomischen Gegebenheiten im relevanten Zeitraum.

Weiter beleuchtet der Autor die Versuche Boliviens, seine Binnenlage durch bilateriale Verhandlungen und durch die Befassung von internationalen Foren mit dieser Problematik zu beheben. Er definiert dabei die Forderung des "returno al mar" auch als ein entwicklungspolitisches Postulat. Die juristische Grundlage, auf die Bolivien seine Position aufbaut, glaubt Hummer nicht exakt qualifizieren zu können, denn er erkennt vier verschiedene Konzeptionen, die einander rechtlich zwar teils widersprechen, aber dennoch nebeneinander von Bolivien vertreten werden.

Die auf über 29 Seiten dargetane geschichtliche Ausgangslage wird jedoch nicht einer eigenen juristischen Wertung unterzogen. Dies ist allein deshalb bedauerlich, weil schon der Titel der Arbeit die Analyse einer Revindikation historischer Gebietstitel verspricht, was allerdings kaum gelingen kann, wenn diese nicht einmal aus dem Geschichtsablauf als juristisch meßbare Größen herauskondensiert werden.

Hummer greift für die juristische Betrachtung, neben der allgemeinen Binnenlageproblematik, das Angebot Chiles vom 19. 12. 1975 heraus, in dem Bolivien die Schaffung eines unter dessen Souveränität stehenden Korridors zum Pazifik in der Gegend von Arica in Aussicht gestellt wurde. In überzeugender Weise stellt er eine derartige Lösung als Zessions- und damit als Sukzessionsproblem dar, versäumt es aber, den Implikationen der Konvention über die Staatennachfolge in Bezug auf völkerrechtliche Verträge von 1978 in dieser Frage nachzuspüren. Der Autor selbst räumt ein, daß die von ihm behandelte Option als Lösungsmöglichkeit der konkreten Forderung Boliviens kaum in Betracht kommt. Wie oben erwähnt, gewährt der Vertrag von Lima Peru ein Vetorecht in dieser Hinsicht, von dem es de facto schon Gebrauch gemacht hat. So gelangt Hummer dann auch zu dem Ergebnis, daß der einzige Weg, der zu einer für alle Seiten tragbaren Lösung führen könnte, über trilaterale Verhandlungen gehen muß, zur "Erarbeitung neuer Vorschläge". Eine Prognose, wie diese neuen Vorschläge aussehen könnten, wagt der Autor nicht.

Zusammenfassend läßt sich sagen: Die historische Darstellung der Problemlage und die treffende Analyse einer Lösungsmöglichkeit dazu verdrängen den revindikatorischen Aspekt, den man im Vordergrund der Arbeit erwartet hätte. Allerdings, so scheint es, 
möchte Hummer dem Leser gerade auch die "undogmatische Art und Weise der Behandlung" der Frage (Einleitung), als ein Beispiel von lateinamerikanischem Pragmatismus in Völkerrechtsfragen vor Augen führen. Kurzum, er möchte in umfassendem Sinne Verständnis für südamerikanische Denkweise in dieser Hinsicht wecken. Ob sich lateinamerikanische Juristen und Historiker allerdings selbst von Hummer verstanden fühlen, wenn dieser von der "totalen Zerstörung des Inkareiches in bewunderungswürdiger Schnelligkeit" spricht, erscheint zweifelhaft.

Marc Weller

\author{
Robert D. Craig/Frank P. King \\ Historical Dictionary of Oceania \\ Greenwood Press, Westport/Conn., London, 1981, XXXVI, 392 S., $\mathfrak{E}$ 47,50
}

106 Autoren behandeln über 300 Stichworte zu Geschichte und Gegenwart, zu Politik, Wirtschaft, Kultur, Religion der Ozeanischen Territorien. Einträge zu geographischen Einheiten, zu den Persönlichkeiten, die durch Entdeckungen und politische Führung prägend waren für die Inseln des Südpazifik, stehen neben solchen, die politische oder historische Schlagwörter erläutern (z. B. "Bismarck Agreement of 1879«), Epochen umreißen (z. B. "German Colonial Empire") oder aktuelle Probleme der Region behandeln (z. B. "Alcohol in the Pacific") - eine pragmatische, rein am Grad des Informierungsinteresses ausgerichtete Auswahl also, die gar nicht erst versucht, die heterogene Region über den Leisten einheitlicher Kategorien zu schlagen.

Das Buch erschließt reiche Information über ein Gebiet, das wohl das derzeit in Europa am wenigsten bekannte der Welt ist, trotz der Penetration durch Portugiesen, Spanier, Briten, Franzosen, Holländer, Russen und Deutsche, trotz Südseeromantik und Paul Gauguin. Zwischen 1840 und dem Zweiten Weltkrieg standen fast alle Inseln und Archipele Ozeaniens unter extrakontinentaler Herrschaft; noch heute bieten die dortigen Hoheitsverhältnisse mehr Anschauungsmaterial für dem Untergang geweihte völkerrechtliche Konzeptionen der Abhängigkeit als jeder andere Teil der Erde. Wie Selbstfindung und eigenständige Entwicklung zustandezubringen sind, wie die Erfahrungen der Abhängigkeit verarbeitet und wie die geeigneten Institutionen entwickelt werden können, um diese Prozesse zu befördern, kommt der auf die großen Kontinente der Dritten Welt gerichteten Sozialwissenschaft bisher kaum in den Blick. Wer dies, von welcher Wissenschaftsdisziplin aus auch immer, ändern will, dem ist dieser Band ein nützliches Handwerkszeug, der durch ein übersichtliches Verweisungssystem, zahlreiche bibliographische Angaben, mancherlei Tabellen und Namenkonkordanzen auch weiteren Einstieg vermittelt.

Die Karten freilich sind nicht mehr als Orientierungsskizzen. Den positiven Gesamteindruck vermögen sie kaum zu schmälern.

David von Vineta 